\title{
Present/absent sample matching in pigeons: Is comparison choice controlled by the sample stimulus or by differential sample responding?
}

\author{
JANICE E. WEAVER, BRIGETTE R. DORRANCE, and THOMAS R. ZENTALL \\ University of Kentucky, Lexington, Kentucky
}

\begin{abstract}
In two experiments involving present/absent sample matching, we tested whether the visual stimuli or differential sample behavior served as the basis for comparison choice. In both experiments, one group (FR/DRO) was required to peck the present sample and to refrain from pecking the absent sample (as typically occurs with fixed duration present/absent samples), and the other group (FR/FR) was required to peck both samples. In Experiment 1, the samples were a black dot on a white field (present) and the white field alone (absent). In Experiment 2, the samples were a yellow hue (present) and a dark response key (absent). In both experiments, divergent retention functions were found only for the FR/DRO group. These results suggest that, in nonhedonic present/absent sample matching, it is the behavior directed toward the present sample, rather than the visual stimulus itself, that serves as the basis for comparison choice.
\end{abstract}

In the typical matching-to-sample task with pigeons, a subject is presented with a choice between two comparisons (e.g., red and green) following the presentation of one of two possible samples (e.g., red or green). After presentation of a red sample, for example, choice of the red comparison is correct; whereas, after presentation of a green sample, choice of the green comparison is correct. Once this task has been acquired, a retention interval (RI) of variable duration can be inserted between the offset of the sample and the onset of the comparisons, to assess memory for the samples. With increasing RIs, the typical finding is that matching accuracy declines at a comparable rate on trials initiated by each sample.

If the samples involve the presence versus the absence of an event, however, the decline in matching accuracy with increasing RI is typically different for trials involving the two sample types. For example, when food and the absence of food (no-food) have been used as samples, matching accuracy on food sample trials has declined rapidly with increasing RI, whereas matching accuracy on no-food sample trials remained high at all values of the RI (see, e.g., Colwill, 1984; Grant, 1991; Sherburne \& Zentall, 1993a, 1993b; Wilson \& Boakes, 1985). But, divergent retention functions are not limited to tasks in which the samples consist of the presence versus the absence of hedonic events. Similar divergent retention functions have been found when samples consist of the presence versus the absence of a hue or a shape (Grant, 1991;

This research was supported by National Science Foundation Grant IBN 9414589 and National Institute of Mental Health Grant 55118 to T.R.Z. We thank Lou Sherburne and Tricia Clement for their contributions to this research. Correspondence concerning this article should be addressed to T. R. Zentall, Department of Psychology, University of Kentucky, Lexington, KY 40506-0044 (e-mail: zentall@pop.uky.edu).
Sherburne \& Zentall, 1993b). Thus, the phenomenon has considerable generality.

The finding of divergent retention function suggests, counterintuitively, that absent samples are remembered better than present samples. However, the fact that matching accuracy on absent-sample trials appears to show no decline with increasing RI and that matching accuracy on present-sample trials typically falls below chance $(50 \%$ correct) has been interpreted as evidence for the asymmetrical coding of samples (see Grant, 1991).

According to the asymmetrical coding hypothesis, only the present sample is coded (or remembered), and responses to the comparison associated with the absent sample occur by default (Colwill, 1984; Grant, 1991; Wilson \& Boakes, 1985). Thus, on present-sample trials, as the RI increases and there is poorer memory for the present sample, it is increasingly likely that a response to the comparison associated with the absent sample will be made. On absent-sample trials, however, there is little decline in matching accuracy with increasing RI, because nothing has been coded (or remembered), so there is nothing to forget. Thus, it has been hypothesized that pigeons have developed a single-code/default strategy (Sherburne \& Zentall, 1993a).

Recent unpublished data from our laboratory indicate that, when pigeons acquire present/absent-sample matching with fixed-duration samples, they tend to respond differentially to the samples, pecking at the sample when it is present, but not when it is absent. For example, when yellow and no-yellow samples were presented for a fixed duration of $2 \mathrm{sec}$, pigeons responded at a rate of 1.34 pecks per second to the yellow samples and at a rate of 0.02 pecks per second to the dark key on the no-yellow sample trials. Similarly, response rates of 1.73 and 0.03 pecks per second were obtained to yellow and no-yellow samples, 
respectively, when the samples were presented for $4 \mathrm{sec}$. Thus, although pecking at the samples is not typically required in present/absent sample matching, there is evidence that it occurs and that it occurs differentially.

The purpose of the present research was to determine whether differential sample responding is necessary to obtain the divergent retention functions typically found in nonhedonic present/absent-sample matching. Specifically we asked whether the divergent retention functions, which are often taken as evidence for the development of a single-code/default-coding strategy, depend on differential responding to the present/absent samples. The coding strategy was assessed by comparing the retention functions of pigeons that were required to respond to the samples differentially with those of pigeons that were required to respond similarly to both samples.

\section{EXPERIMENT 1}

In Experiment 1, the sample response requirement was manipulated between groups, so that, for one group, the pigeons were required to peck the present sample and to refrain from pecking the absent sample (a pattern of responding typically found when pigeons match present/ absent samples of fixed duration), whereas for two other groups, pecking was required to both present and absent samples.

Pigeons in the FR/DRO group were required to peck the present sample (a black dot located in one quadrant of a white field) 20 times (fixed ratio, FR 20) but to refrain from pecking the absent sample (a white field with no black dot). The procedure for this group was intended to mimic the pattern of behavior generally found in present/ absent matching tasks when no response is required but pecking generally occurs to the present sample. It was assumed that pigeons in this group could use either the presence of the dot or responding as the basis of a single code.

Pigeons in the FR/FR group were required to peck both the present sample and the absent sample on an FR 20 schedule. They were required to peck both samples, to ensure that the behavior in the presence of both samples was similar and could not be used as the basis for comparison choice.

If the present-sample code is based solely on the visual aspects of the present sample, both groups should show divergent retention functions. Alternatively, if differential sample responding is necessary for asymmetrical coding, divergent retention functions should be obtained only for the FR/DRO group. To the degree that the present sample code is based on responding to the present sample (but not the absent sample), the divergence in retention functions should be reduced or eliminated for the FR/FR group (i.e., this group should show parallel retention functions).

\section{Method}

\section{Subjects}

The subjects were 12 White Carneaux pigeons purchased as retired breeders (7-9 years old) from Palmetto Pigeon Plant (Sumter,
SC). All the subjects had had experience with simultaneous discrimination tasks. The pigeons were individually housed in wire cages, with free access to water and grit. The colony room was maintained on a 12:12-h light:dark cycle. All the subjects were maintained at or above $80 \%$ of their free-feeding weights with mixed grain provided mainly in experimental sessions.

\section{Apparatus}

The experiment was conducted in a sound-attenuated threeresponse-key operant chamber (LVE/BRS, Laurel, MD). The chamber measured $35 \mathrm{~cm}$ high $\times 30.5 \mathrm{~cm}$ wide $\times 35 \mathrm{~cm}$ across the response panel. The three round pecking keys were $2.54 \mathrm{~cm}$ in diameter and were horizontally aligned on the response panel, $6.5 \mathrm{~cm}$ apart center to center, with the bottom edge of the keys being $19 \mathrm{~cm}$ above the grid floor. Mounted behind each key was a 12-stimulus in-line projector (Industrial Electronics Engineering, Series 10, Van Nuys, CA, with General Electric No. 1820 lamps). The center key could be illuminated with a white field (no-dot) and with a white field with one of four black dots. The black dots measured $5 \mathrm{~mm}$ in diameter and were positioned in one of the four quadrants defined by the vertical and horizontal axes of the center key. The projectors for the side keys projected red and green fields (Kodak Wratten filters Nos. 26 and 60, respectively). Access to a rear-mounted grain feeder filled with Purina Pro grains was provided through a horizontally centered opening in the response panel $(6 \times 5 \mathrm{~cm})$. The bottom edge of the feeder was located $7 \mathrm{~cm}$ above the grid floor. A feeder lamp was illuminated whenever the grain feeder was operated. A shielded houselight, centered on the ceiling of the chamber, $13 \mathrm{~cm}$ back from the response panel, provided general illumination during the intertrial interval (ITI). Masking noise was provided by an exhaust fan and by white noise delivered through a speaker mounted on the back of the response panel. The experiment was controlled by a microcomputer located in an adjacent room.

\section{Procedure}

Training. Because all of the pigeons had had experience with a simultaneous discrimination, they were immediately placed on zero-delay matching-to-sample training. The response requirement to the sample was increased gradually over sessions from FR 1 to FR 20 for the samples to which pigeons were required to peck (all the samples except dot-absent samples for pigeons in the FR/DRO group). Pigeons in the FR/DRO group $(n=4)$ were required to peck the dot sample, which appeared at the same location on the response key on all the present trials on an FR 20 schedule, and to refrain from pecking the no-dot sample for $6 \mathrm{sec}$ (DRO $6 \mathrm{sec}$ ). The duration of the DRO schedule was selected to approximate the time required to complete the FR 20 schedule. Pigeons in the FR/FR group ( $n=8$ ) were required to peck both the present (dot) and the absent (no-dot) samples on an FR 20 schedule. For half of the pigeons in the FR/FR group $(n=4)$, on present-sample trials, the dot appeared at the same location on the response key on all the presentsample trials (the FR/FR-one group). For the remaining birds ( $n=4$ ), the dot appeared randomly at the top left, top right, bottom left, and bottom right, equally often in each session (the FR/FR-many group). This manipulation was included in order to ensure that the pigeons attended to the dot on the key and not to some idiosyncratic aspect of the stimulus pattern. For pigeons in the FR/DRO and FR/FR-one groups, the location of the dot was constant on all present-sample trials, but the dot position was counterbalanced over subjects.

For half of the pigeons in each group, choice of the red comparison was correct following a dot sample, and choice of the green comparison was correct following a no-dot sample. For the remaining birds, this assignment was reversed. Correct comparison choices resulted in 2-sec access to mixed grain at the start of the 10-sec ITI. Incorrect comparison choices were followed by the ITI only. Experimental sessions were conducted once a day, 6 days per week. Each session consisted of 96 trials (48 trials of each sample type), with comparison position counterbalanced across trials. 
The pigeons that showed a position or hue preference in training (more than 90 of 96 choices per session of either one key position or one hue, following a minimum of 10 sessions on task) were given correction training consisting of repeat trials (a maximum of five per trial) following an error. Training on zero-delay matching continued until a criterion of $90 \%$ correct or higher on each sample type was maintained for 2 consecutive sessions or until an alternative criterion of at least 8 sessions with $83 \%$ correct or higher on both sample types was met.

Retention test. Testing began on the session following completion of the training criterion. Mixed RIs of $0,1,2$, and $4 \mathrm{sec}$ (Test Set 1) were inserted between sample offset and comparison onset. Each RI duration occurred equally often for each trial type (12 trials at each duration for each sample type for the FR/DRO and FR/FRone groups and, to allow for complete counterbalancing of the position of the dot, 16 trials at each duration for dot and no-dot samples for the FR/FR-many group). No stimuli were presented during the dark RI. Following 10 sessions on Test Set 1 RIs, delays were doubled to $0,2,4$, and $8 \mathrm{sec}$ (Test Set 2). Test Set 2 continued for 10 sessions.

\section{Results and Discussion}

One subject in the FR/FR-many group was dropped because of experimenter error. Analysis of the acquisition and test data from the FR/FR-one versus the FR/FRmany groups indicated that these groups did not differ in the number of sessions required to reach criterion $(F<1)$. The .05 level of significance was adopted for all the analyses. A three-way mixed factor analysis of variance (ANOVA), performed on the test data for the two FR/FR groups, with group, sample type, and RI as factors, yielded no significant main effect of group or interactions involving group. Thus, the data from these two groups were combined, and the combined group is referred to as the FR/FR group in all further analyses.

\section{Training}

Pigeons in the FR/DRO group acquired the zero-delay matching task to criterion in $12(S E M=1.13)$ sessions, whereas pigeons in the FR/FR group required 72.4 $(S E M=11.15)$ sessions to meet criterion. The difference in acquisition between the two groups was significant $[F(1,9)=13.65]$. Relatively rapid acquisition by the FR/ DRO group demonstrates the beneficial effects of differential sample responding (see, e.g., Zentall, Hogan, Howard, \& Moore, 1978). Apparently, the dot/no-dot sample discrimination was particularly difficult when the sample response requirement was the same for both samples.

\section{Retention Test: Set 1}

To avoid ceiling effects that might obscure differences in matching accuracy between sample types, only those sessions on which the pigeons' overall performance was below $90 \%$ correct were used in analysis of the RI data. Thus, the following analyses included an average of 8.75 ( $S E M=0.41)$ sessions per subject for the FR/DRO group and $9.86(S E M=0.13)$ sessions per subject for the FR/ FR group.

The retention functions for the FR/DRO group on the first retention test were clearly divergent (see the left panel of Figure 1), as is typical of present/absent-sample trial retention functions. However, no such divergence was found for the FR/FR group (see the right panel of Figure 1). For this group, the present/absent-sample trial retention functions were parallel and were virtually identical.

A three-way mixed factors ANOVA, with group, sample type, and RI as factors, was performed on the RI data (pooled across sessions). The analysis revealed a significant group $\times$ sample type $\times \mathrm{RI}$ interaction $[F(3,27)=$
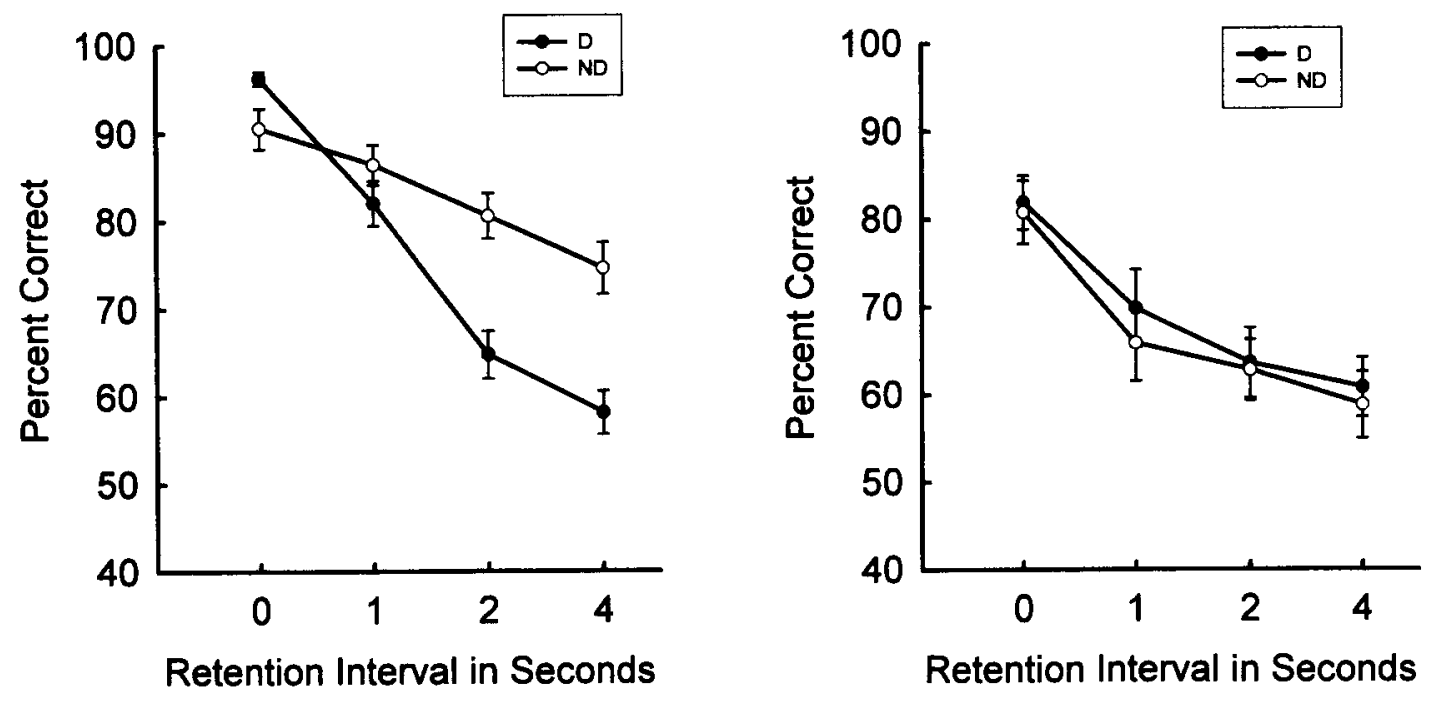

Figure 1. Percent correct as a function of retention interval on dot (D, filled circles) and no-dot (ND, open circles) sample trials for the FR/DRO group (left panel) and the FR/FR group (right panel) for Test Set 1. 
3.28]. The interaction indicates that the sample type $\times$ RI interaction differed between groups. The analysis also revealed a significant effect of group $[F(1,9)=7.74]$, indicating that overall performance was somewhat higher for the FR/DRO group than for the FR/FR group. It appears that the superiority of differential responding was maintained during testing.

Separate two-way analyses were conducted for each group. For the FR/DRO group, the sample type $\times$ RI interaction was significant $[F(3,9)=5.95]$, as was the effect of RI $[F(3,9)=54.18]$. For the FR/FR group, however, only the effect of RI was significant $[F(3,18)=19.21]$.

\section{Retention Test: Set 2}

Retention functions for the second retention test were similar to those from the first test. Analyses included an average of $8.5(S E M=0.98)$ sessions for subjects in the FR/DRO group and all $10(S E M=0)$ sessions for subjects in the FR/FR group. The retention functions for data from the FR/DRO group were even more divergent than those for the first test (see the left panel of Figure 2). In fact, at the longest delay, present-sample-trial matching accuracy actually dropped below chance. For the FR/FR group, once again, the retention functions were essentially equivalent (see the right panel of Figure 2). A three-way mixed factors ANOVA similar to that performed on the Set 1 data revealed a significant group $\times$ sample type $\times$ $\mathrm{RI}$ interaction $[F(3,27)=9.07]$, indicating that the sample type $\times$ RI interaction differed as a function of group.

Again, separate two-way analyses were conducted for each group. An analysis of the data from the FR/DRO group indicated that there was a significant sample type $\times \mathrm{RI}$ interaction $[F(3,9)=19.46]$, consistent with the typically found divergent retention of present/absent samples. Analysis of the data from the FR/FR group revealed no significant sample type $\times \mathrm{RI}$ interaction $[F(3,18)=$
1.47]. Again, only the effect of RI was significant $[F(3,18)=13.44]$.

The strongly divergent retention functions found for the FR/DRO group suggest that this group was using a single-code/default strategy. That is, they coded the dot sample and responded to the comparison associated with the no-dot sample by default. More importantly, however, the performance of the FR/FR group indicates that differential sample responding may be a necessary condition for asymmetrical coding. Similar retention functions found for each sample type in the FR/FR group suggests that those pigeons were coding each sample independently, despite the fact that the pigeons in this group could have developed a default response to the no-dotassociated comparison and coded only the visual presence of the dot. Apparently, presence of the dot versus its absence does not lead invariably to a single-code/default strategy. Dot present versus dot absent was chosen as the samples in Experiment 1 so that pigeons in the FR/FR group would readily learn to peck both samples. It may be, however, that such samples differ in important ways from the present/absent samples typically used in this research (e.g., yellow and the absence of yellow-a dark key). Thus, in Experiment 2 we attempted to replicate this design, using more typical present/absent samples.

\section{EXPERIMENT 2}

In Experiment 2, the present sample consisted of a yellow field, and the absent sample consisted of a dark response key (i.e., the absence of the yellow field). Again, the pigeons in the FR/FR group were required to peck each sample, to ensure that behavior in the presence of both sample types was similar and that the use of responding as the basis for the present sample code was precluded. As in Experiment 1, the pigeons in the FR/DRO group were re-
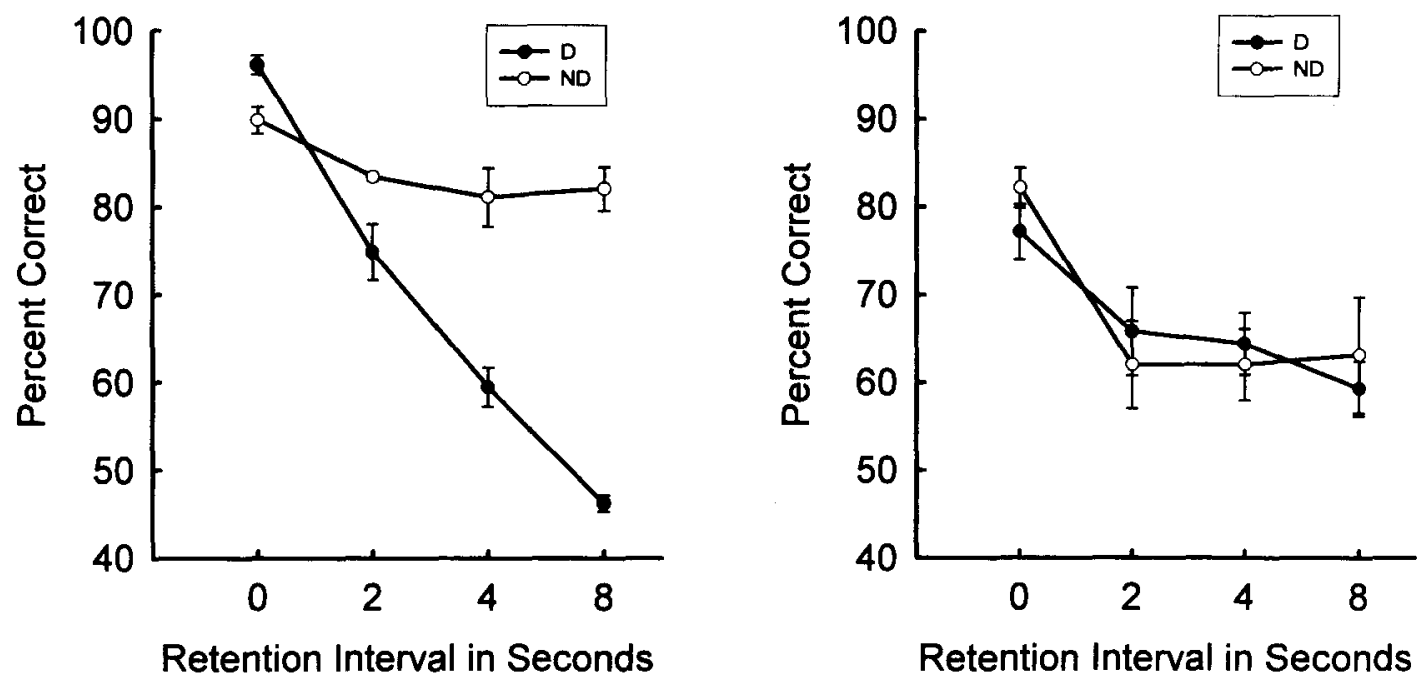

Figure 2. Percent correct as a function of retention interval on dot (D, filled circles) and no-dot (ND, open circles) sample trials for the FR/DRO group (left panel) and the FR/FR group (right panel) for Test Set 2. 
quired to peck the yellow sample and to refrain from pecking the no-yellow sample, to mimic the behaviors generally displayed toward samples of this type. If differential sample behavior toward the present and the absent sample is a requirement for asymmetrical coding and, thus, for the divergent retention functions typically found following present/absent-sample-matching training, it was hypothesized that divergent retention functions would be found for the FR/DRO group, but not for the FR/FR group.

Method
Subjects
The subjects were 16 pigeons similar in type and experience to
those used in Experiment 1. The pigeons were maintained in the
same manner as were those in Experiment 1.

\section{Apparatus}

The operant chamber was similar to the one used in Experiment 1 , with the following exceptions. The chamber measured $31.5 \mathrm{~cm}$ high, $32 \mathrm{~cm}$ wide, and $35.5 \mathrm{~cm}$ across the front panel. The response keys were square $(2.5 \times 2.5 \mathrm{~cm})$ and were placed $4 \mathrm{~cm}$ apart, with their bottom edges located $20 \mathrm{~cm}$ above the grid floor. The chamber contained two houselights. One houselight, horizontally centered in the middle of the response panel, $6 \mathrm{~cm}$ above the response keys, was illuminated during all the samples. The second houselight, which was centered on the ceiling $12 \mathrm{~cm}$ from the response panel, was illuminated during the ITI. The yellow field (Kodak Wratten filter No. 9) could be projected onto the center response key by a rear-mounted in-line projector. All other aspects of the operant chamber were the same as those used in Experiment 1.

\section{Procedure}

Pretraining. The pigeons in the FR/FR group $(n=8)$ were hand shaped by the method of successive approximations to peck the dark center key (absent sample) when the response panel houselight was on. Once pigeons reliably pecked during the absent sample, they were placed on task. The pigeons in the FR/DRO group $(n=8)$ were placed on task without pretraining.

Training. Training was similar to that in Experiment 1, except for the following. Only five responses were required to the two sam- ples for the FR/FR group and to the FR sample for the FR/DRO group. For the FR/DRO group, the pigeons were required to refrain from responding to the absent (dark key) sample for $2.5 \mathrm{sec}$ (DRO $2.5 \mathrm{sec}$ ). No repeat trials were used in Experiment 2. Training on zero-delay matching continued to a criterion of $90 \%$ correct for two consecutive sessions.

Retention test. The retention test was identical to that used for Test Set 1 in Experiment 1, except that it consisted of only eight test sessions.

\section{Results and Discussion}

\section{Training}

The pigeons in the FR/DRO group acquired the task in an average of $9.8(S E M=1.66)$ sessions, and pigeons in the FR/FR group in an average of $14.6(S E M=2.83)$ sessions. A one-way ANOVA revealed that, because of considerable variability in learning rates, this difference in sessions to criterion was not significant $[F(1,14)=1.99]$.

\section{Retention Test}

Again, sessions with performance over $90 \%$ correct were removed from consideration, to avoid ceiling effects that might obscure differences in matching accuracy between trial types for each group. The following analyses are based on an average of $7.75(S E M=0.15)$ and $7.5(S E M=0.35)$ sessions per subject in the FR/ DRO and FR/FR groups, respectively. Retention functions for the FR/DRO group were again divergent. The retention function on no-yellow sample trials was relatively flat with increasing RI, whereas the retention function on yellow sample trials was relatively steep (see the left panel of Figure 3). In contrast, for the FR/FR group, retention functions for the two sample types were relatively similar (see the right panel of Figure 3 ).

A three-way mixed factors ANOVA was performed on the data, with group, sample type, and RI as factors. The analysis revealed that there was a significant group $\times$
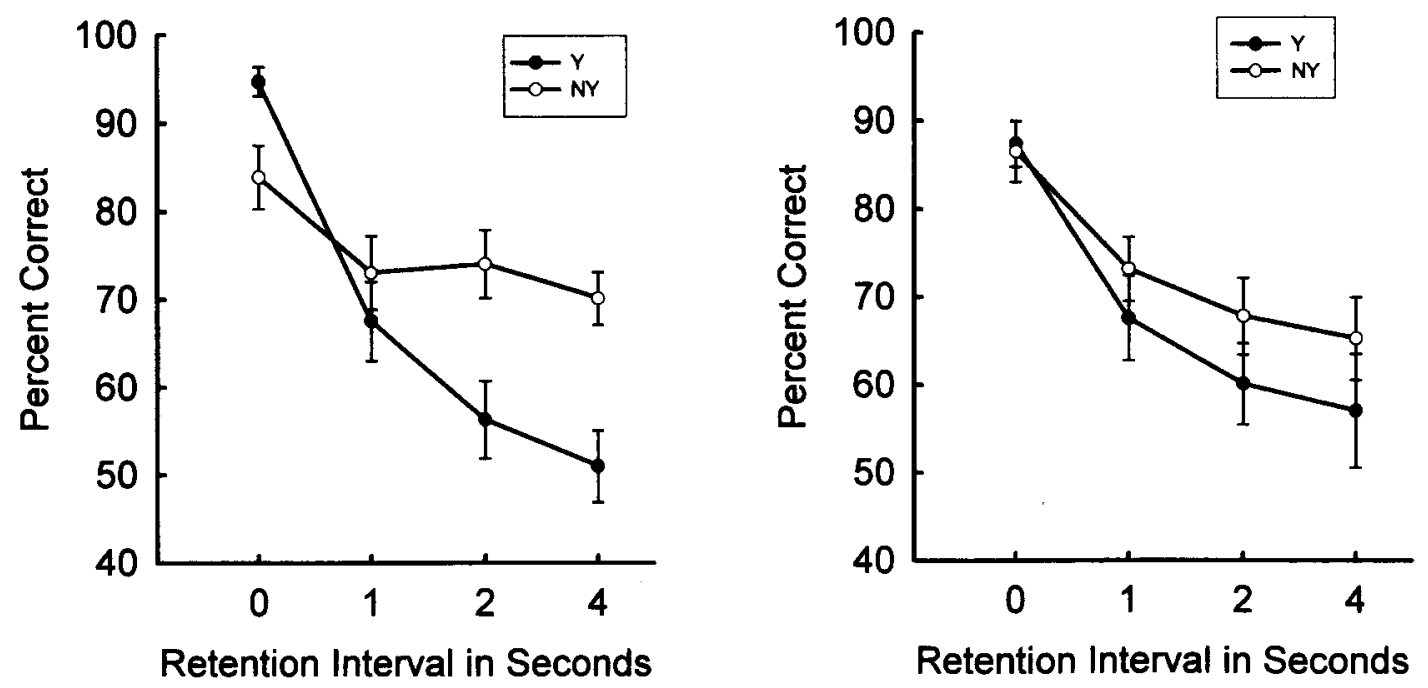

Figure 3. Percent correct as a function of retention interval on yellow (Y, filled circles) and no-yellow (NY, open circles) sample trials for the FR/DRO group (left panel) and the FR/FR group (right panel). 
sample type $\times \mathrm{RI}$ interaction $[F(3,42)=2.88]$, indicating that there was greater divergence of the retention functions for the FR/DRO group than for the FR/FR group.

Separate two-way ANOVAs were performed for each group. For the FR/DRO group, the sample type $\times$ RI interaction was significant $[F(3,21)=11.12]$, whereas for the FR/FR group, it was not. For the FR/FR group, only the effect of RI was significant $[F(3,21)=80.74]$. Although it appears that matching accuracy on no-yellow sample trials was superior to that on yellow sample trials for the FR/FR group, neither the sample type $\times$ RI interaction $[F(3,21)=1.08]$ nor the main effect of sample type $(F<1)$ was significant.

The data from Experiment 2 with hue/no-hue samples replicate the results of Experiment 1 . Again, significantly divergent retention functions were obtained for only the FR/DRO group. The relatively parallel retention functions found on yellow and no-yellow sample trials for the FR/FR group are consistent with the notion that nondifferential responding to present/absent samples does not result in divergent retention functions.

\section{GENERAL DISCUSSION}

The present research was motivated by the observation that, when pigeons are trained on a present/absent-samplematching task involving samples of a fixed duration, with no responding required to the samples, they typically peck at the present sample and refrain from pecking at the absent sample. The purpose of the present research was to determine whether such differential sample responding is necessary to obtain the divergent retention functions that have been interpreted as evidence for the development of a single-code/default-coding strategy. In Experiment 1 , dot versus no-dot samples were used, and divergent retention functions were found for the group trained to respond differentially to the samples, but not for the group trained to respond similarly to both samples. In Experiment 2, a more typical present/absent-sample task was used, involving the presence versus the absence of a yellow sample, and again, divergent retention functions were found only for the group trained to respond differentially to the samples. These results suggest that asymmetrical coding (as assessed by divergent retention functions) does not occur in the absence of differential sample responding.

An exception to this rule regarding present/absentsample matching may be the case of food and its absence as samples. Although pigeons do, in fact, peck at food and not at its absence, Sherburne and Zentall (1993a) have suggested that either food or responding can serve as the basis for the single-code/default strategy. Sherburne and Zentall trained pigeons on a food-present/absent-samplematching task but included trials involving a red sample to which responding was required and which was associated with the same comparison as the no-food sample. Thus, because the pigeons pecked at both the food and the red sample, differential sample responding could not easily be used as the basis for comparison choice. Nevertheless, these pigeons showed divergent sample retention functions on food-sample versus red-sample trials. These data suggest that food (rather than pecking) can serve as the basis for a single-code/default strategy. Further support for this hypothesis comes from the data from another group in this experiment, in which the pigeons were required to refrain from responding to the red sample and the correct comparison was the same as that for the food sample. Thus, for this group, neither food nor pecking could be used consistently as the basis for comparison choice. Consistent with the present hypothesis, retention functions for this group were not divergent, suggesting that a single-code/default strategy did not develop.

Although Sherburne and Zentall (1993a) suggest that pigeons can show some flexibility in what serves as the basis for the single code in hedonic present/absent-samplematching tasks (e.g., either the presence of food or pecking), in general (i.e., with nonhedonic present/absent samples), the visual attributes of the samples are not sufficient to generate a single-code/default strategy in the absence of differential sample responding. In nonhedonic present/ absent-sample matching, it appears that the present sample is coded as pecking, despite the fact that, in general, the samples are presented for a fixed duration and responding is not required.

There are two possible explanations for the absence of divergent retention functions when pigeons are required to peck at both the present and the absent samples. First, it is possible that requiring the pigeon to peck at the absent sample causes that sample to be coded (e.g., as dark key sample) and a single-code/default strategy is no longer used. In other words, the samples change from eventnonevent, when pecking is not required to the dark key, to event-event, when pecking is required to both samples.

Alternatively, it is possible that the introduction of delays following training without delays produces ambiguous conditions during test (see Zentall, 1997). For example, if during training, a pigeon had learned to choose comparison stimuli on the basis of whether it had just been pecking or not, the introduction of delays (during which it, presumably, would not be pecking) might result in response competition. That is, in the case of a presentsample trial, the absence of pecking during the delay might compete with the earlier sample-pecking response, and furthermore, the longer the delay, the greater might be the tendency to choose the comparison associated with not pecking (i.e., the comparison associated with the absent sample). In the case of an absent-sample trial, however, the absence of pecking during the delay would be consistent with the earlier absence of pecking the absent sample, and not only should there be no response competition, but, in addition, there should be no loss of matching accuracy with increasing RI. This is exactly the pattern of results that has been reported.

When responding is required to both samples (as is the case with the FR/FR groups in the present experiments), however, differential responding to the samples cannot 
be used as the basis for comparison choice. Thus, differential response competition should not occur, and parallel retention functions should result. Although the results of the present experiments do not allow one to conclude that such response competition is responsible for the divergent functions typically found when delays are introduced following training with present/absent-sample matching, they do suggest that the role of the absence of responding during the RI in such tasks should be examined further.

\section{REFERENCES}

CoLwILL, R. M. (1984). Disruption of short-term memory for reinforcement by ambient illumination. Quarterly Journal of Experimental Psychology, 36B, 235-258.

Grant, D. S. (1991). Symmetrical and asymmetrical coding of food and no-food samples in delayed matching in pigeons. Journal of Experimental Psychology: Animal Behavior Processes, 17, 186-193.
Sherburne, L. M., \& Zentall, T. R. (1993a). Asymmetrical coding of food and no-food events by pigeons: Sample pecking versus food as the basis of the sample code. Learning \& Motivation, 24, 141-155.

Sherburne, L. M., \& Zentall, T. R. (1993b). Coding of feature and no-feature events by pigeons performing a delayed conditional discrimination. Animal Learning \& Behavior, 21, 92-100.

WILSON, B., \& BOAKES, R. A. (1985). A comparison of the short-term memory performances of pigeons and jackdaws. Animal Learning \& Behavior, 13, 285-290.

ZENTALL, T. R. (1997). Animal memory: The role of "instructions." Learning \& Motivation, 28, 280-308.

Zentall, T. R., Hogan, D. E., Howard, M. M., \& Moore, B. S. (1978). Delayed matching in the pigeon: Effect on performance of samplespecific observing responses and differential delay behavior. Learning \& Motivation, 9, 202-218.

(Manuscript received June 3, 1998; revision accepted for publication March 16, 1999.) 